\title{
Rethinking the Magnetic Properties of Lepidocrocite: A Density Functional Theory and Cluster Expansion Study
}

\author{
Daniel J. Pope and Aurora E. Clark \\ Department of Chemistry, Washington State University, Pullman, Washington 99164, USA \\ Micah P. Prange and Kevin M. Rosso \\ Pacific Northwest National Laboratory, Richland, Washington 99532, USA
}

(Dated: February 23, 2020)

\begin{abstract}
The iron oxyhydroxide lepidocrocite $(\gamma-\mathrm{FeOOH})$ is an abundant mineral critical to a number of chemical and technological applications. Of particular interest is the ground state and finite temperature magnetic order, and the subsequent impact this has upon crystal properties. The magnetic properties, investigated in this work are governed primarily through superexchange interactions, and have been calculated using density functional theory and cluster expansion methods. Quantification of these exchange terms has facilitated the determination of the ground state magneto-crystalline structure and subsequent calculation of its lattice constants, elastic moduli, cohesive enthalpy, and electronic density of states. Further, using a collinear magnetic configuration model, the magnetic heat capacity versus temperature has been studied and the Néel temperature obtained.
\end{abstract}

\section{INTRODUCTION:}

Iron oxides are a common class of minerals whose applications span an array of disciplines, from biotechnology, to environmental science, to electronics. Lepidocrocite, $\gamma$-Fe(III)OOH is a naturally occurring iron oxy-hydroxide that is most common in rocks, soils, and rusts. 1] While it has wide general relevance, its industrial application derives from its high temperature thermal decomposition to maghemite, 2] whose thin films are ubiquitous in magnetic storage, transistors, and other modern electronics applications. 3 .

The magnetic properties of lepidocrocite have been studied extensively, 4 8 but there remain discrepancies between the experimentally measured behavior and what might be expected from this class of mineral. The magnetochemistry of iron oxides is governed primarily by the superexchange interactions. 9 Superexchange provides an effective antiferromagnetic coupling across the Fe sublattice. Hence, if superexchange is the dominant effect, an antiferromagnetic ordering is expected for the ground state. The magnitude of exchange defines the magnetic coupling strength and is heavily dependent on the Fe-O-Fe bond angles and distances. Iron oxides often exhibit Néel/Curie temperatures that are commensurate with their respective exchange parameters as determined by these Fe-O-Fe bonds. 10] Due to the strength of superexchange in these systems, magnetic disorder typically manifests at temperatures above $800 \mathrm{~K}$ in the oxides, and around 300K for the oxy-hydroxides. 1] The Néel temperature for lepidocrocite, however, is estimated to be between 50 and 77K. 4, 6] This is a surprisingly low value given its crystal structure, in which double chains of edge-sharing $\mathrm{FeO}_{6}$ octahedra along the [001] direction are connected by edge-sharing along [001] to comprise layers in its orthorhombic unit cell.[11] These layers are stacked along [010] and connected by hydrogen bonding.
In contrast to $\alpha-\mathrm{FeOOH}$ (goethite) and $\beta-\mathrm{FeOOH}$ (akaganeite), whose structures consist of double chains of iron octahedra connected by corner-sharing, 1] the bulk structure of lepidocrocite is comprised of two-dimensionally periodic edge-sharing ( $\mathrm{Fe}-\mathrm{O}-\mathrm{Fe}-\mathrm{O}$ )- bonding interactions along both its $a$ and $c$ axes. Given this, it is reasonable to predict that magnetic order to be maintained at higher temperatures than its polymorphic counterparts.

A number of theories have been presented to account for the experimentally measured low temperature transition of lepidocrocite. These include amorphous crystallinity, 4] the presence of water impurities, [5] or small concentrations of other magnetic ion inclusions. 6, 12. One of the most common explanations is that the measured low temperature magnetic disorder is due to superparamagnetism arising from the nanoscale domain sizes in lepidocrocite platelets used in experiment. 5 . 7 Evidence for higher temperature magnetic order in bulk lepidocrocite has been determined to be consistent with a model of uncompensated surface magnetic moments present in small particles. 8, 13. Yet this study also conclude that superparamagnetism is due to the presence of small diameter maghemite phases, maintaining that the Néel temperature of lepidocrocite is between 50 and $77 \mathrm{~K}$. It is, however, important to note that no characteristic peak in the magnetic susceptibility measurements was observed, indicating no phase transition to a disordered state. This is corroborated by heat capacity measurements, where no magnetic phase transition peaks are observed up to temperatures of $400 \mathrm{~K}$, at which lepidocrocite then undergoes dehydration to form maghemite. 14

In this work we seek to better define the magnetic interactions between $\mathrm{Fe}(\mathrm{III})$ in lepidocrocite, which we accomplish using density functional theory in combination with cluster expansion methods to compute the ground state magneto-crystalline unit cell. Using these meth- 
ods, we determined the different strengths of magnetic coupling pairs as detailed in Sec. IIIA As a result, we propose a new ground state magneto-crystalline unit cell symmetry in Sec. IIIB, one that matches experimental data well and improves upon the magnetic descriptions used in previous computational literature. 15 18] Using this structure, we then recalculate a host of various properties of lepidocrocite and show improved agreement with respect to experiment. In Sec. IIIG, finite temperature predictions from Monte Carlo simulations using the results from cluster expansion are discussed. Our findings are summarized in Sec. IV]

\section{THEORY AND METHODOLOGY:}

\section{A. Magnetic Modeling Methods}

A physically complete theoretical description of magnetism in condensed matter is enormously challenging. By itself, first principles determination of the ground state magnetic unit cell, which can be larger than that for the atomic structure, is not immediately straightforward. Finite temperature properties require surmounting even larger hurdles. In this work we approach magnetic properties through approximation of the real system by a collinear disordered local moment (DLM) model.19. Although noncollinear, magnon, and spin-wave effects are ignored, thermodynamic properties have successfully been calculated for a number of systems using this simplified model. 20,24]

In practice, our model system entails a combination of local spin-up and spin-down configurations creating some stoichiometric relation of $\mathrm{Fe}(\uparrow)_{x} \mathrm{Fe}(\downarrow)_{1-x} \mathrm{OOH}$ in the bulk. By assuming these colinear states are eigenstates, the total energy of the system can then be represented by Eq. (1):

$$
E(\sigma)=J_{0}+\sum_{j<i} J_{i j} \sigma_{i} \sigma_{j}+\sum_{k<j<i} J_{i j k} \sigma_{i} \sigma_{j} \sigma_{k}+\cdots
$$

where $\sigma_{i}= \pm 1$ denotes the sign of the projection onto an arbitrary quantization axis of the electronic spin at site $i$ (the projection is assumed to have magnitude $5 \hbar / 2$ since we expect 5 unpaired electrons of the same spin at each $\mathrm{Fe}$ due to large intra-atomic exchange effects).

Using cluster expansion methods [25], as implemented in the Alloy Theoretical Automated Toolkit (ATAT), the $J_{i j}$ of Eq. (1) can be represented as the effective cluster interactions (ECIs) of an alloy of spin-up and -down sites. Ideally, these ECIs represent the interaction energies for all possible cluster sizes and configurations and are exact for the complete basis. They are determined by solving the set of linear equations relating the total energy of a configuration $\mathrm{E}$ to the cluster interaction energy $J$ through the cluster correlation term $\prod$, where $\prod$ is related to the probability of finding some cluster pattern $\alpha$ in some configuration $\sigma$, and $\mathrm{E}$ is calculated by $a b$ initio methods. This series of interactions is truncated to some limit of clusters that give a reasonable cross validation score, $(<0.025)$, a result of predicted energies being within reasonable agreement of calculated energies for a series of configurations.

With a given set of ECIs, the predicted energy of any configuration can be rapidly calculated. There are two benefits to this. First is the ability to rapidly search all possible ground state configurations up to reasonable supercell sizes. This is valuable for determining the magnetic order of anti-ferromagnetic or ferrimagnetic systems in anisotropic crystal structures, where all possible configurations within even small supercell sizes would be unfeasible to calculate directly. These predicted energies can then be verified with $a b$ initio calculations. The second is the ability to determine thermodynamic properties through Monte Carlo methods. In this manner the magnetic disorder can be estimated through calculation of heat capacity vs. temperature curves, where the magnetic phase transition occurs as some peak in this curve.

\section{B. Computational Details}

Electronic structure calculations were performed using the Vienna ab initio simulation package (VASP) 26. within the framework of plane-wave density functional theory in periodic boundary conditions, using the projector augmented wave (PAW) 27. method to represent the core electrons. Due to the large number of calculations required for determination of ECI's, the exchange correlation is represented by the computationally inexpensive generalized gradient approximation $(\mathrm{GGA}+\mathrm{U})$ using the Perdew, Burke, and Ernzerhof (PBE) 28 variant. A U-J Hubbard correction value of $4 \mathrm{eV}$ was used, consistent with prior computational work of Fe(III) oxides. 2931] The crystal structure is described using the $\mathrm{A} 2{ }_{1}$ am space group (not $\mathrm{Cmc} 2_{1}$ or $\mathrm{Cmcm}$ ). In $\mathrm{A} 2_{1} a m b$, not $c$, is the stacking direction. Unit cell parameters and fractional atomic coordinates were simultaneously varied in the energy relaxation. Calculations for using the PBE0 functional 32 were performed to verify 0K properties independent of the Hubbard $\mathrm{U}$ parameter. The plane wave basis energy cutoff for all structures was converged to energy differences of less than $1 \mathrm{meV}$ per $50 \mathrm{eV}$ increase. For all systems, the maximum required energy cutoff, $900 \mathrm{eV}$, was used across all systems to ensure accurate energy comparisons. To avoid Pulay stress error, the energy cutoff was increased by $30 \%$ for all geometry optimizations. A static calculation at the original cutoff was then performed for to obtain the total energy. Brillouin zone sampling was performed with gamma-centered k-points using the Monkhorst-Pack scheme 33 and again converged to energy differences within $1 \mathrm{meV}$ for all unit cell crystal structures with a k-point mesh of $6 \times 2 \times 6$. All supercell structures were sampled inversely proportional to their size in each cell dimension.

Finite temperature properties were determined 
through Monte Carlo simulations of a 6000 iron atom simulation cell using ATAT's Easy Monte Carlo Code (EMC2). 34 Equilibrium ensemble energies were determined for the temperature range of 0 to $1000 \mathrm{~K}$ at $1 \mathrm{~K}$ intervals. At each interval, 5,000 equilibration steps were performed followed by 50,000 production run steps. Heat capacities were then determined from finite differences centered over a range of $10 \mathrm{~K}$.

Vibrational energy contributions were determined from frozen phonon mode calculations with anharmonicity effects estimated using the quasi-harmonic approximation. VASP was used to calculate the dynamical matrix of the AF system at a series of volumes about the equilibrium volume. Thermodynamic properties were then calculated using phonopy. 35. The constant pressure vibrational heat capacity was calculated with the quasiharmonic approximation using the calculated values of the bulk modulus and thermal expansion coefficient.

\section{Calculated Lattice Properties}

Lattice constants were determined from the cell geometry optimization from VASP using an energy cutoff of $10^{-4} \mathrm{eV}$ between steps. The bulk modulus was calculated by fitting the Birch-Murnaghan equation of state to the energy-volume dependence. Elastic moduli were calculated using the stress-strain method as implemented in VASP.

The experimental cohesive enthalpy is the measured heat of formation of the crystal product per formula unit (f.u.) minus the reactant gas phase components. The calculated cohesive enthalpy uses the total energy calculated for the crystal minus the energies of the gas phase components, according to:

$$
\begin{aligned}
\Delta H_{f}= & E\left(M \uparrow_{1-x} M \downarrow_{x} O O H\right) \\
& -(1-x) E(M \uparrow)-x E(M \downarrow) \\
& -2 E(O)-E(H)
\end{aligned}
$$

\section{RESULTS AND DISCUSSION:}

\section{A. Effective Cluster Interactions}

The J's of Eq. (1) are given by the Effective Cluster Interactions (ECI's) between the Fe(III) spins. The estimated total energies of configurations using the ECI's were compared with ab initio calculations resulting in an excellent cross validation score of $3.8 \times 10^{-4}$, confirming the model's predictive power. While multi-site interactions of $\mathrm{Fe}(\mathrm{III})$ clusters of size three and greater were calculated, only pairwise interactions were found to have contributions to the total energy greater than $1 \mathrm{meV}$. Table I details the properties of all pair interactions with energies greater than $1 \mathrm{meV}$ and are visually represented in Fig. 2. Pairs 2 and 3 are both AF edge-sharing irons

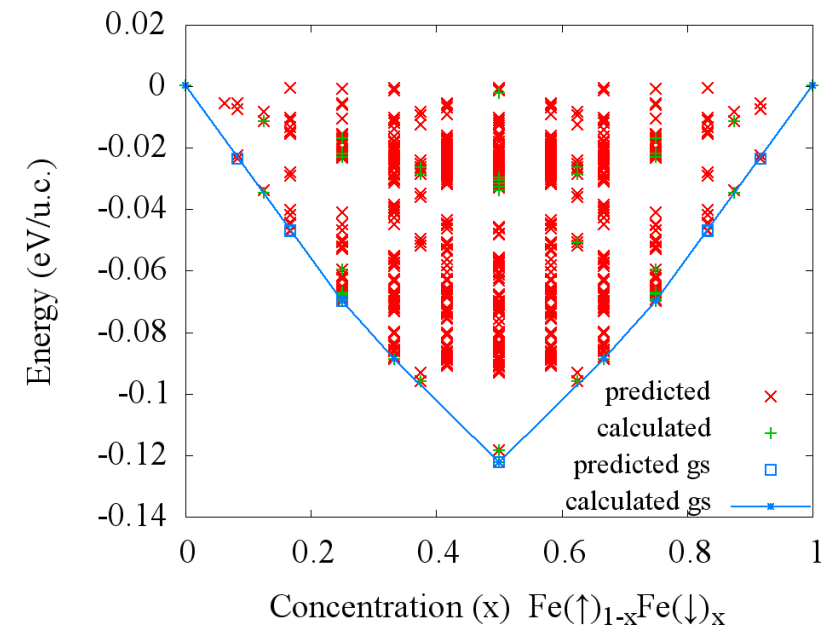

(a)

(b)

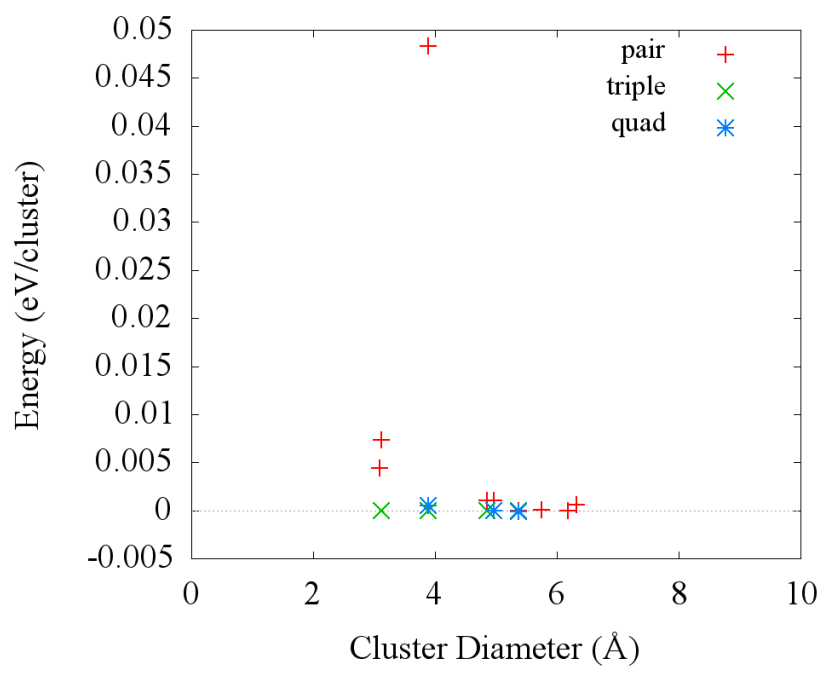

FIG. 1. Top: total energy per unit cell from Eq. 11) (red) and DFT simulations (green) for a variety of spin configurations $\left\{\sigma_{i}\right\}$ vs. the fraction $x$ of spin-up sites. The lowest energy for each $x$ from Eq. [1] is marked with blue squares; DFT simluations of the same same clusters are connected with blue lines. Bottom: The magnitude of interaction energies for the largest $J_{i j}$ (red), $J_{i j k}$ (green), $J_{i j k l}$ (blue) in $\mathrm{eV}$ vs the largest atom pair distance in the cluster.

with weak interactions likely due to the effect of near $90^{\circ}$ Fe-O-Fe bond angles on superexchange. The strongest interaction is AF pair 4 oriented along the c-axis. The $\mathrm{Fe}-\mathrm{O}-\mathrm{Fe}$ bond angle of $157^{\circ}$, the closest bond angle to $180^{\circ}$ in the system, greatly contributes to the superexchange interaction. These findings are consistent with experimental work relating AF strength to Fe-O-Fe bond angle. [10] Pairs 5 and 6 are the weakest interactions, occurring over large Fe-Fe distances within the crystal. Although weak, the multiplicity of the interactions would still contribute significantly to the total energy. 
TABLE I. Effective Cluster Interactions (ECIs)

\begin{tabular}{lcccc}
\hline \hline Fe(III) pair & multiplicity & ECI $(\mathrm{meV} /$ pair $)$ & distance $(\AA)$ & Fe-O-Fe angle $\left({ }^{\circ}\right)$ \\
\hline 2 & 4 & 4.4 & 3.09 & 98.2 \\
3 & 8 & 7.3 & 3.12 & 97.7 \\
4 & 4 & 48.3 & 3.89 & 157 \\
5 & 4 & 1.1 & 4.85 & - \\
6 & 8 & 1.0 & 4.97 & - \\
\hline \hline
\end{tabular}

(a)

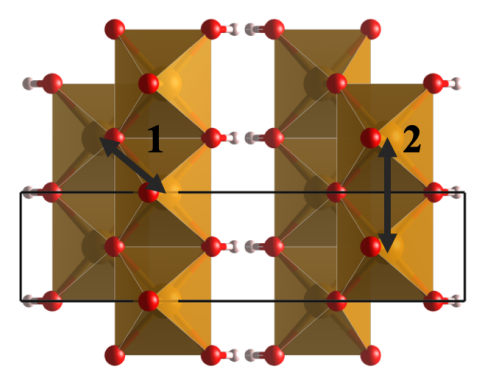

(b)

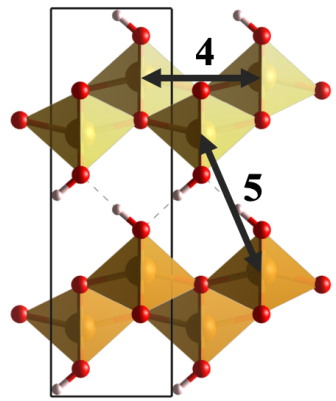

(c)

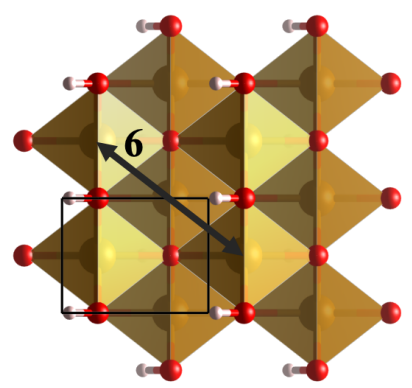

FIG. 2. All pairwise interactions with significant ECI's

B. Magnetic Order:

To determine the ground state magnetic order, configurations of increasing supercell size were exhaustively searched by ATAT, and the ECI's were used to quickly predict the energies of these systems. Discovered lower energy configurations were then confirmed with ab initio calculations.

The lowest energy structure found was a net zero magnetic moment AF system. Its structure departs from prior computational work, in that the crystallographic symmetry is broken by the inclusion of magnetic order, resulting in the smallest repeating unit of a $2 \times 1 \times 2$ supercell of the conventional cell. In the conventional cell, Fig. 3 (a), the system is restricted to FM ordering along the $a$ and $c$ axes. Expansion to the supercell allows for the AF ordering along these same axes Fig. 3(b). Of special significance is the allowed AF ordering along the c-axis, where the Fe-O-Fe bonds are near $180^{\circ}$. Here, pair interactions are strongest, leading to a large decrease in total energy. Exclusion of this strong interaction would likely have an impact on calculations of this system. Also of note is the predicted presence of AF layering, as described in the experimental literature. 10] This layering occurs diagonally to the primary crystal axes in strips oriented between the a and c axes and perpendicular to the (010) surface as shown in Fig. 3.(c).

Given the new predicted magneto-crystalline structure, it is of interest to calculate how the crystal properties may differ from those based upon the conventional cell. In the following sections we compare calculated values for the newly found lowest energy structure, referred to as AF, with those of the lowest energy structure found having the conventional cell symmetry, AF'.

\section{Density of States}

The density of states (DOS) is sensitive to the magnetic order of a material resulting in possible changes to peak shapes and band gap sizes. Band gaps previously calculated for lepidocrocite, on the basis of the conventional unit cell, have been used to determine the U-J Hubbard term used in GGA+U calculations by fitting to measured band gaps of, for example, 2.06eV.[1] However, such experimental measurements are typically performed at room temperature, and it is unclear what the magnetic disorder is at these temperatures and how it may influence band gap measurements. Shown in Fig. 4, there is a significant difference between the calculated DOS of AF and AF'. AF displays much sharper peaks and a significantly larger band gap of $0.5 \mathrm{eV}$.

\section{Lattice Parameters}

Calculated lattice parameters for the $\mathrm{AF}$ and $\mathrm{AF}$ ' structures are shown in Table III and are compared to experimental values. Lattice parameters were taken to be the cell dimensions of the crystallographic unit cells comprising the 2x1x2 supercell. Notably, all cell parameters in the ground state structure are within $1 \%$ of experiment, an improvement relative to previous studies. 15, 17, 18, The errors become larger for the AF' structure, indicating the importance of choosing the correct ground-state magneto-crystalline cell. In particular, a large reduction in error occurs along the $c$-axis entailing the near $180^{\circ} \mathrm{Fe}-\mathrm{O}-\mathrm{Fe}$ magnetic interaction. There is also an especially good match along the $b$-axis where lepidocrocite is layered by hydrogen bonded sheets. This could possibly be due to two reasons. Deformation along 
(a)

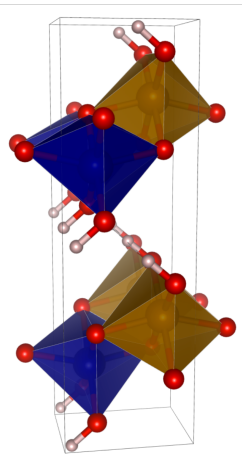

(b)

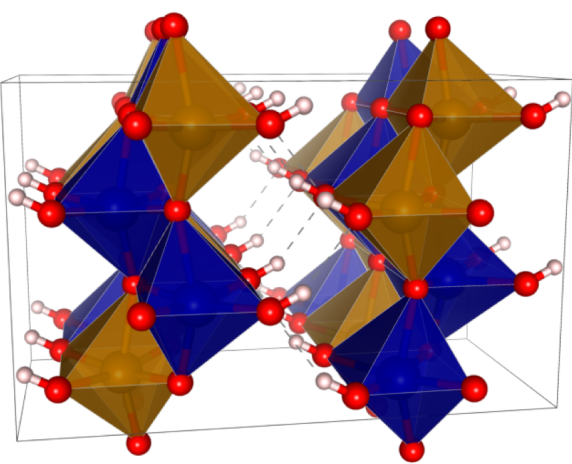

(c)

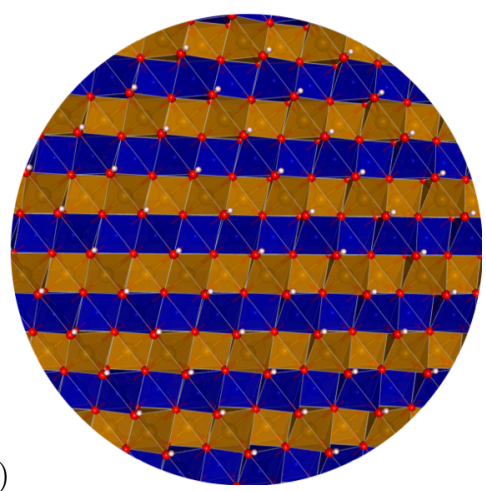

FIG. 3. Antiferromagnetic ordering in lepidocrocite, where Fe $\uparrow \mathrm{Fe} \downarrow$ are represented by blue and yellow and the crystal c-axis is perpendicular to the plane of the paper. Figures (a) AF' (b) AF Figure (c) AF layering on the 010 surface

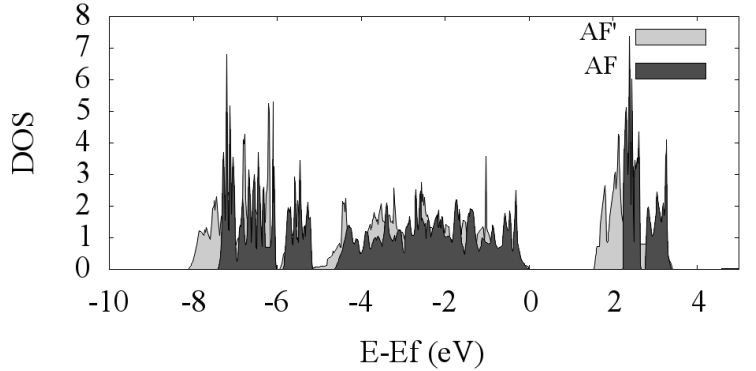

FIG. 4. Total electronic density of states of AF and AF'

the $b$-axis may have an especially weak impact on the total energy due to relatively soft hydrogen bonding interactions connecting the layers. This would allow the interlayer magnetic interactions, though weak, to become impactful on equilibrium distances. Moreover, the $c$-axis spacing may also be correlated with the interlayer distance. Finally, it is noteworthy that the improvement of lattice parameter fits using the $\mathrm{AF}$ ordered system may indicate that the magnetic order of bulk lepidocrocite is preserved at room temperature. Although not practical to implement during the full ATAT analysis, the lattice parameters for the AF system were recalculated using the PBE0 functional and were found to match closely those found using GGA+U.

\section{E. Elastic Constants}

To the best of our knowledge, experimentally measured elastic constants have not been reported. They are also not commonly reported in the computational literature. In Table II we compare the calculated lattice constants for the $\mathrm{AF}$ system to these previous computational studies. For the bulk modulus, the Birch-Murnaghan EOS fit to the energy vs. volume curve is shown in Fig. 5 . The calculated bulk modulus is in good agreement with a result by Otte. 31. Although this work did not defini- tively state the magnetic order of their ground state, the agreement suggests use of the correct ground state structure. In contrast, the bulk modulus is more than twice that reported by Guo, 17] where only configurations of crystallographic unit cell AF systems were considered. This is reflective of a broadening of the energy vs volume curve for the AF system when including the proper pair interactions. The elastic moduli were calculated directly from VASP and also show large differences from those computed for the crystallographic cell. A large difference between the $c_{33}$ values is present as might be expected when comparing cells consisting of AF pairs vs. FM pairs along this axis. More surprisingly, the largest difference is between $c_{22}$ moduli, indicating more compressibility of the hydrogen bonded stacking layers in the AF system. These large differences in elastic properties may provide an additional basis to confirm the the bulk magnetic properties of lepidocrocite at higher temperatures. It may prove especially useful that the largest difference occurs along the axis perpendicular to the large basal surface of lepdicrocite platelets, which often allows deposits of this material to self-orient accordingly.

\section{F. Energetics}

The cohesive enthalpies determined for both $\mathrm{AF}$ and $\mathrm{AF}^{\prime}$ as per Eq. (2) are significantly different by $0.1 \mathrm{eV} /$ (f.u.). However, although in generally good agreement with experiment, the lower energy AF structure has a larger deviation. It should be noted that the experimental value is calculated from standard enthalpies of formation, as a $\Delta \mathrm{H}$ for lepidocrocite extrapolated to $0 \mathrm{~K}$ is unavailable. Therefore it is inconclusive from the energetics calculations which system is more representative of the real system. 
TABLE II. The calculated elastic moduli in units of GPa

\begin{tabular}{lcc}
\hline & & $\mathrm{AF}$ \\
\hline $\mathrm{B}_{0}$ & $74.8^{\mathrm{a}}, 158.9^{\mathrm{b}}$ & 158.6 \\
$\mathrm{c}_{11}$ & $246.9^{\mathrm{a}}$ & 223.6 \\
$\mathrm{c}_{22}$ & $272.2^{\mathrm{a}}$ & 111.4 \\
$\mathrm{c}_{33}$ & $347.7^{\mathrm{a}}$ & 233.5 \\
\hline \hline
\end{tabular}

${ }^{\mathrm{a}}$ Reference [17]. ${ }^{\mathrm{b}}$ Reference [31].

TABLE III. The calculated lattice constants $a, b, c$, and volume in comparison to experimental data.

\begin{tabular}{lcccccc}
\hline \hline Method & Structure & $a$ & $b$ & $c$ & $V$ & CohE \\
\hline & & $(\AA)$ & $(\AA)$ & $(\AA)$ & $\left(\AA^{3}\right)$ & $(\mathrm{kJ} / \mathrm{mol} /$ f.u. $)$ \\
\hline Exp.(293K) ${ }^{\mathrm{a}}$ & & 3.07 & 12.53 & 3.88 & 37.3 & 1683 \\
PBE & AF' & 3.08 & 12.61 & 3.93 & 38.2 & 1717 \\
& $\%$ error & $0.03 \%$ & $0.64 \%$ & $1.30 \%$ & $2.27 \%$ & $2.0 \%$ \\
& AF & 3.10 & 12.53 & 3.90 & 37.86 & 1726 \\
& \% error & $0.81 \%$ & $0.04 \%$ & $0.61 \%$ & $1.47 \%$ & $2.5 \%$ \\
PBE0 & AF & 3.08 & 12.53 & 3.86 & 148.8 & \\
& $\%$ error & $0.30 \%$ & $0.02 \%$ & $0.62 \%$ & $0.07 \%$ & \\
\hline \hline
\end{tabular}

${ }^{\mathrm{a}}$ Reference [1].

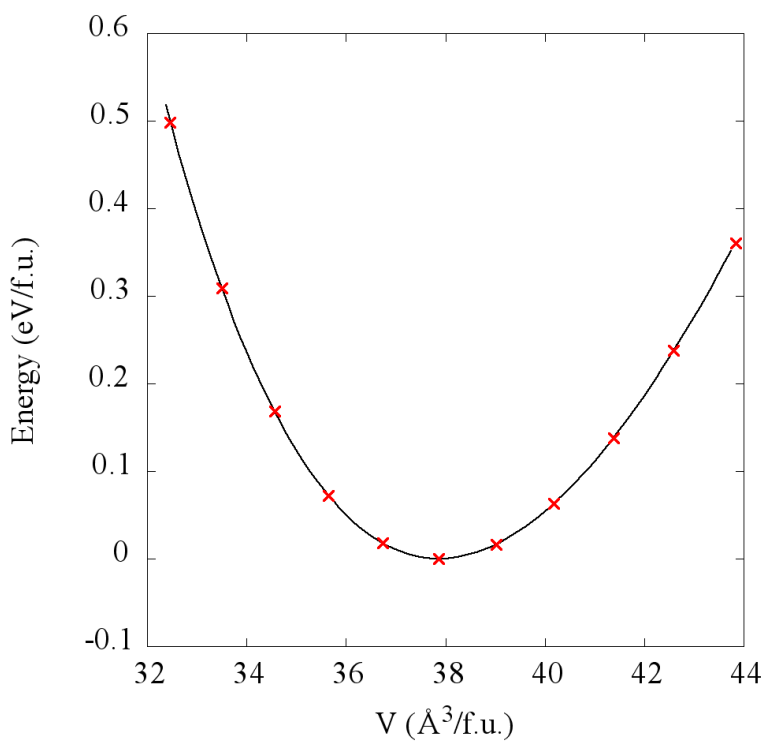

FIG. 5. Energy as a function of volume of the AF system in terms of formula units fit with Birch-Murnaghan EOS. Energy scaled in reference to energy of equilibrium volume.

\section{G. Magnetic Disorder}

The onset temperature of magnetic disorder in lepidocrocite was determined through examination of the heat capacity curve generated from MC calculations. Here the critical temperature was determined as the sec- ond order phase transition identifiable as the anomalous "bump" in the plot of heat capacity vs. temperature, as can be seen in Fig. 6. The $\mathrm{T}_{\mathrm{N}}$ was determined to be $450 \mathrm{~K}$ from the peak in the heat capacity curve. The shape of the calculated curve is representative of a system with Short Range Order (SRO), wherein the exchange interactions continue to contribute to the heat capacity above the critical temperature. This value is much higher than the typical experimentally measured values of $50-77 \mathrm{~K}$, but is very close to the calculated value of $392 \mathrm{~K}$ as determined by Guyodo. 8] In that work, the lepidocrocite particle magnetization was treated as system of uncompensated surface spins plus bulk anti-ferromagnetic contributions, and the critical temperature was determined from mean field calculations from a series of susceptibility measurements at different temperatures. That study concludes that the high temperature bulk AFM properties are due to maghemite inclusions. However this is inconsistent with the much higher critical temperature of maghemite measured between the ranges of 820 to 986K. [1]

The magnetic heat capacity calculations are compared to experimental heat capacity curves in Fig. 7, 36 Notably absent in the experimental data is the existence of any peak indicative of a phase transition. The calculated contributions to the total heat capacity are comprised of the vibrational and magnetic portions. Electronic contributions were omitted due to the large band gap, precluding occupancy of LUMO bands at low temperatures. The addition of the calculated magnetic heat capacity to the total curve matches well with the experimental data. 


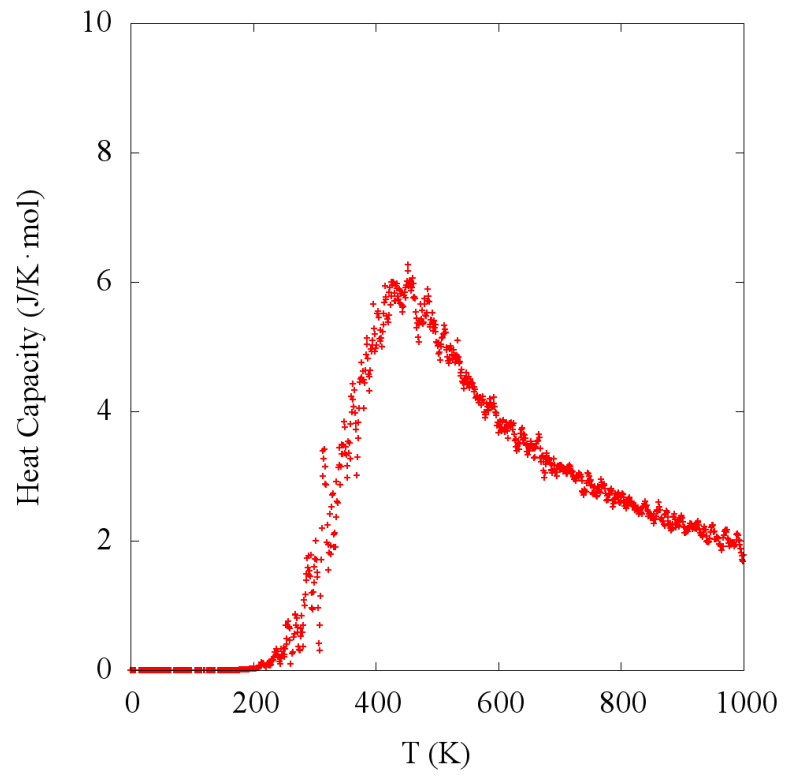

FIG. 6. Heat capacity vs. Temperature as determined from Monte Carlo simulations. Peak corresponding to magnetic order/disorder phase transition occurs at $450 \mathrm{~K}$

However, it is not conclusive in capturing the magnetic disorder. Anharmonicty errors in the phonon mode calculations at high temperatures may be on the order of the differences between the experimental data and the calculated vibrational contribution. There is also no consideration of phonon mode coupling to the magnetic order.

This may be a significant contribution given the effect of magnetic order on the elastic moduli. Additionally, the experimental data stops at $400 \mathrm{~K}$, below the predicted critical point. A much more convincing argument could be made if a peak were captured in experimental data, as other DLM magnetic studies have successfully captured critical temperature peaks for magnetic disorder in heat capacity calculations. Further complicating matters is the onset of a phase transition from lepidocrocite to maghemite at 250K. 37. It is, however, noteworthy that the experimentally observed structural phase transition overlaps with the calculated onset of magnetic disorder. This introduces the interesting prospect that these phenomena are coupled.

\section{CONCLUSIONS:}

We have used density functional theory coupled with cluster expansion methods to determine Heisenberg parameters for all significant cluster interactions within a lepidocrocite crystal having high spin $\mathrm{Fe} \uparrow_{x} \mathrm{Fe} \downarrow_{1-x} \mathrm{OOH}$ stoichiometry aligned parallel to the c-axis. These parameters were then used to determine a ground state AF magnetic order, which was found to be consistent with experiment, but requiring a larger supercell of the conventional cell, a finding that therefore supersedes

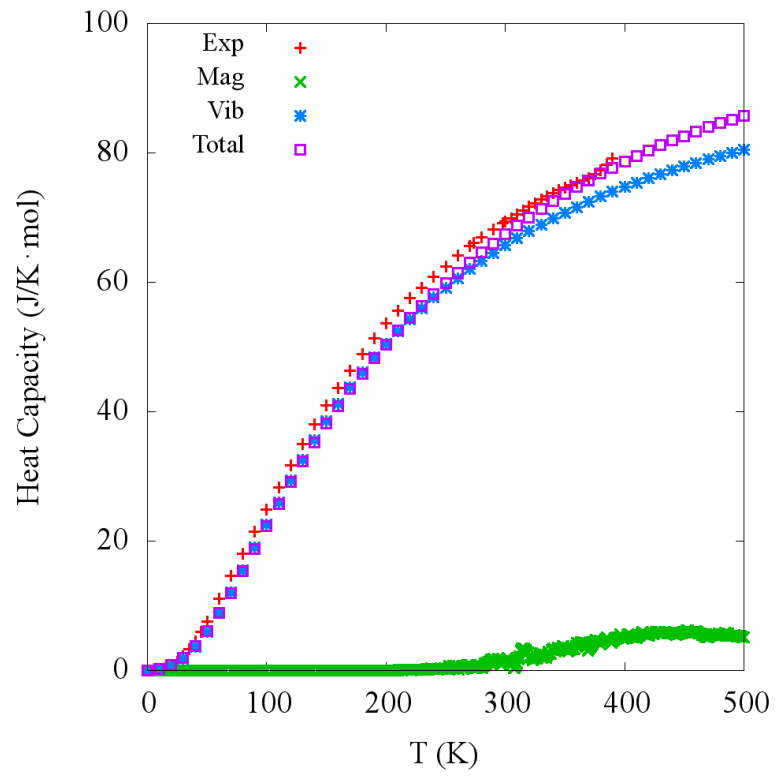

FIG. 7. Experimental (crosses) 36 total heat capacity and calculated (squares) total heat capacity. Calculated total heat capacity is the sum of the vibrational (x's) and magnetic heat capacity (stars).

prior computational work. Lattice properties of this new ground state structure were calculated and found to yield improvements in the lattice constants, and significant differences in the elastic moduli, cohesive enthalpy, and electronic DOS.

Magnetic thermodynamics properties were determined through Monte Carlo simulations of the Heisenberg model system. A Néel temperature of $450 \mathrm{~K}$ was determined, consistent with the strength of the calculated lepidocrocite magnetic exchange interactions. This may provide further evidence that the low experimentally measured temperature of lepidocrocite is indeed due to superparamagnetism of fine domain sizes within particles. The calculated total heat capacity matches well with experiment, but the absence of a characteristic peak in the experimental heat capacity data makes confirmation of the calculated critical temperature difficult.

\section{ACKNOWLEDGMENTS}

This work was supported by IDREAM (Interfacial Dynamics in Radioactive Environments and Materials), an Energy Frontier Research Center funded by the U.S. Department of Energy (DOE), Office of Science, Office of Basic Energy Sciences (BES). Computational work was performed in part at the Center for Institutional Research Computing at Washington State University and using supercomputing resources at the Environmental Molecular Sciences Laboratory (EMSL), a national scientific user fa- 
cility at Pacific Northwest National Laboratory (PNNL). EMSL is sponsored by the Department of Energys Office of Biological and Environmental Research. PNNL is a multi-program national laboratory operated for DOE by Battelle Memorial Institute under Contract DE-AC0576RL0-1830.
[1] R. M. Cornell and U. Schwertmann, The iron oxides: structure, properties, reactions, occurrences and uses (John Wiley \& Sons, 2003).

[2] H. Shokrollahi, J. Magn. Magn. Mater. 426, 74 (2017).

[3] R. Dronskowski, Adv. Funct. Mater. 11, 27 (2001).

[4] C. Johnson, J. Phys. C: Solid State Physics 2, 1996 (1969).

[5] E. De Grave, G. Da Costa, L. Bowen, U. Schwertmann, and R. E. Vandenberghe, Clays Clay Miner. 44, 214 (1996).

[6] A. Hirt, L. Lanci, J. Dobson, P. Weidler, and A. Gehring, J. Geoph. Re.: Solid Earth 107, EPM (2002).

[7] G. Lee, S. Kim, B. Choi, S. Huh, Y. Chang, B. Kim, J. Park, and S. Oh, J. Korean Phys. Soc. 45, 1019 (2004).

[8] Y. Guyodo, P. Bonville, J. L. Till, G. Ona-Nguema, F. Lagroix, and N. Menguy, Front. Earth Sci. 4, 28 (2016).

[9] R. L. Carlin and A. J. van Duyneveldt, Magnetic properties of transition metal compounds (Springer, 1977).

[10] J. Coey, Magnetic properties of iron in soil iron oxides and clay minerals, in Iron in soils and clay minerals (Springer, 1988) pp. 397-466.

[11] F. Ewing, J. Chem. Phys. 3, 420 (1935).

[12] J. Till, Y. Guyodo, F. Lagroix, G. Ona-Nguema, and J. Brest, Earth Planet. Sci. Lett. 395, 149 (2014).

[13] C. Gilles, P. Bonville, K. Wong, and S. Mann, Eur. Phys. J. B - Condens. Matter Complex Syst. 17, 417 (2000).

[14] J. Majzlan, K.-D. Grevel, and A. Navrotsky, Am. Min. 88, 855 (2003).

[15] K. M. Rosso and J. R. Rustad, Am. Min. 86, 312 (2001).

[16] N. Pinney, J. D. Kubicki, D. S. Middlemiss, C. P. Grey, and D. Morgan, Chem. Mat. 21, 5727 (2009).

[17] H. Guo and A. S. Barnard, Phy. Rev. B 83, 094112 (2011).

[18] V. Alexandrov and K. M. Rosso, J. Chem. Phys. 140, 234701 (2014).

[19] I. A. Abrikosov, A. Ponomareva, P. Steneteg, S. Baran- nikova, and B. Alling, Curr. Opin. Solid State Mater. Sci. 20, 85 (2016).

[20] Y. Wang, L. Hector Jr, H. Zhang, S. Shang, L. Chen, and Z. Liu, Phys. Rev. B 78, 104113 (2008).

[21] S.-L. Shang, Y. Wang, and Z.-K. Liu, Phy. Rev. B 82, 014425 (2010).

[22] S.-L. Shang, J. E. Saal, Z.-G. Mei, Y. Wang, and Z.-K. Liu, J. Appl. Phys. 108, 123514 (2010).

[23] B. Alling, T. Marten, and I. A. Abrikosov, Nat. Mater. 9, 283 (2010).

[24] B. Alling, T. Marten, and I. Abrikosov, Phys. Rev. B 82, 184430 (2010).

[25] A. van de Walle, Methods for first-principles alloy thermodynamics, JOM 65, 1523 (2013).

[26] G. Kresse and J. Furthmüller, Phy. Rev. B 54, 11169 (1996).

[27] G. Kresse and D. Joubert, Phys. Rev. B 59, 1758 (1999).

[28] J. P. Perdew, K. Burke, and M. Ernzerhof, Phys. Rev. Lett. 77, 3865 (1996).

[29] G. Rollmann, A. Rohrbach, P. Entel, and J. Hafner, Phys. Rev. B 69, 165107 (2004).

[30] I. Leonov, A. Yaresko, V. Antonov, M. Korotin, and V. Anisimov, Charge and orbital order in f e 3 o 4, Phys. Rev. Lett. 93, 146404 (2004).

[31] K. Otte, R. Pentcheva, W. W. Schmahl, and J. R. Rustad, Phys. Rev. B 80, 205116 (2009).

[32] C. Adamo and V. Barone, J. Chem. Phys. 110, 6158 (1999).

[33] J. D. Pack and H. J. Monkhorst, Phy. Rev. B 16, 1748 (1977).

[34] A. van de Walle and M. Asta, Model. Simul. Mater. Sci. Eng. 10, 521 (2002).

[35] A. Togo and I. Tanaka, Scr. Mater. 108, 1 (2015).

[36] R. J. Lemire, U. Berner, C. Musikas, D. A. Palmer, P. Taylor, O. Tochiyama, and J. Perrone, Tech. Rep. (Data Bank, 2013).

[37] A. Gehring and A. Hofmeister, Clays Clay Miner. 42, 409 (1994). 